\title{
Mechanical characterisation of TiN/ZrN multi-layered coatings
}

\author{
C.J. Tavares ${ }^{\mathrm{a}, *}$, L. Rebouta ${ }^{\mathrm{a}}$, M. Andritschky ${ }^{\mathrm{a}}$, S. Ramos ${ }^{\mathrm{b}}$ \\ ${ }^{a}$ Depto. Física, Universidade do Minho, Azurém, 4800 Guimarães, Portugal \\ ${ }^{\mathrm{b}}$ Depto. Eng ${ }^{a}$ Mecânica (polo 2), FCT da Univ. de Coimbra, Pinhal de Marrocos, 3030 Coimbra, Portugal
}

\begin{abstract}
Ultra-microhardness, adhesion and residual-stress analysis tests were performed on reactive sputtered deposited TiN/ZrN multi-layers. Hardness values as high as $~ 3600$ Vickers were achieved for this material. Scratch tests of coatings deposited on steel substrates confirmed the existence of different mechanisms associated with total adhesion failure, depending essentially on multi-layer deposition control parameters. Stress-relaxation measurements indicated the compressive nature of these thin films. The inherent mechanical characterisation was broadened regarding the induced contributions from film thickness, total interfacial roughness, number of bi-layers and corresponding modulation periodicity. Complementary analyses with data extracted from structural XRD studies have been undertaken. (C) 1999 Elsevier Science S.A. All rights reserved.
\end{abstract}

\section{Introduction}

Multi-layers are one-dimensional synthetic structures comprising a number of alternate layers. They fit in a vast range of optical, electrical/magnetical and protection against wear applications. Therefore, the physical properties of multi-layers have been the subject of considerable interest due to the fact that the diverse phenomena associated with very thin films, interfaces, roughness and anomalous mechanical properties, can be studied. It has been reported that multi-layer coatings may be superior to single-layer coatings regarding particular applications [1-3]. Nevertheless, a systematic study concerning their failure mechanisms has yet to be established.

Hard wear physical vapour deposited (PVD) coatings have now reached a new generation. The alternative multi-layer design is credited to the introduction of more interfaces, which permit cracks to be deflected, thereby dissipating energy and enhancing toughness. Recently it was reported that multi-layers are suited for tribological applications owing to their elevated hardness and strength [4]. Others have commented that multi-layers have superior hardness, due either to differences in dislocation line energies or coherency strain fields developed between adjacent

*Corresponding author. Tel.: +351-53-510154; fax: +351-53-510153 E-mail address: ctavares@ fisica.uminho.pt (C.J. Tavares) layers [5]. Moreover, the modulation period of the multilayer is known to induce an effect on the overall microhardness of such coatings, this being possibly a synergistic improvement [6].

This paper reports the mechanical characterisation of TiN/ZrN multi-layer thin films grown by PVD. Ultra-microhardness, adhesion and residual-stress analyses have been undertaken. The deposition of these coatings was achieved by unbalanced reactive magnetron sputtering, combining RF and DC modes.

\section{Fabrication of the samples}

The TiN/ZrN coatings were deposited using an Alcatel SCM650 sputtering system. An $\mathrm{Ar} / \mathrm{N}_{2}$ enriched atmosphere was present in the chamber. Two types of multi-layers were fabricated: in the static mode, alternating layers of TiN and $\mathrm{ZrN}$ were grown by stopping the substrate holder during a preset time interval alternately above the $\mathrm{Ti}$ and $\mathrm{Zr}$ targets; in the rotation mode the substrate holder rotates continuously (for a specific cycle period) between both targets. The Ar flow rate was kept at $100 \mathrm{~cm}^{3} / \mathrm{min}$ whilst the $\mathrm{N}$ flow rate was fixed at $2.25 \mathrm{~cm}^{3} / \mathrm{min}$ in the static mode and $4.25 \mathrm{~cm}^{3} / \mathrm{min}$ in the rotation mode. Pure $\mathrm{Ti}$ and $\mathrm{Zr}$ targets were used. The $\mathrm{Ti}$ target was run in the RF mode $(600 \mathrm{~W}$ in static and $1200 \mathrm{~W}$ in rotation) whilst that corresponding to $\mathrm{Zr}$ was in the $\mathrm{DC}$ 
mode $(0.9 \mathrm{~A}$ in static and $1.0 \mathrm{~A}$ in rotation). The substrate bias voltage was held at $-50 \mathrm{~V}$. The target-to-substrate distance was kept at $60 \mathrm{~mm}$ in all depositions.

The samples were grown on $40 \mathrm{~mm}$ diameter high-speed steel (AISI M2) discs. These substrates were previously heat-treated and annealed to enhance their overall dynamic hardness. Also, prior to loading in the sputtering chamber, the substrates were fine polished and cleaned through a sequence consisting in sandpaper-to-diamond paste abrasion, ultrasonically-enhanced alkali decreasing and washing stages, followed by de-ionised water rinsing, and finally, hot air drying. Before deposition, the substrates were in situ sputter etched in Ar, with the RF mode (200 W), for $15 \mathrm{~min}$.

The examination of the cross-section of these coatings with a scanning electron microscope (SEM) provided their thickness.

\section{Mechanical characterisation}

In this section will be described the most important formalism which resulted in the measurement of the ultra-microhardness, Young's modulus, critical loads and residual stress.

\subsection{Ultra-microhardness measurements}

Hardness testing has achieved the status of one of the simplest and quickest experimental techniques by means of which to obtain information regarding aspects of mechanical properties. Recent developments in hardness testing have enabled continuous monitoring of the load and depth of penetration experienced by the indenter during the indentation process. These depth-sensing measurements allow the estimation not only of the hardness but also of Young's modulus.

The ultra-microhardness experiments were carried out on a computerised Fischerscope dynamic ultra-microhardness tester (model H100). This set-up enables continuous monitoring of the load $(P)$ and penetration depth $(h)$ during the indentation cycle. The tester was equipped with a quadrangular pyramidal Vickers indenter, possessing an apical angle of $136^{\circ}$. The approach is to estimate the residual plastic indentation depth $\left(h_{\mathrm{p}}\right)$ remaining after indentation unloading [7]. This value of $h_{\mathrm{p}}$ undergoes an error correction due to geometric defects at the indenter's tip (off-set), yielding consequently $h_{\mathrm{pc}}$. Therefore, the equation that enables the calculation of the Vickers hardness (HV) in $\mathrm{kg} / \mathrm{mm}^{2}$ is given by

$\mathrm{HV}=\frac{P}{240.25 h_{\mathrm{pc}}^{2}}$.

The denominator stands for the characteristic area function of the Vickers indenter [8]. In order to evaluate Young's modulus, one has to determine the initial unloading contact stiffness $(S)$ :

$S=\frac{\mathrm{d} P}{\mathrm{~d} h}$.

This is actually the slope of the initial portion of the unloading curve. Finally, the following is obtained [8]:

$S^{-1}=\frac{\mathrm{d} h}{\mathrm{~d} P}=\frac{1}{2 h_{\mathrm{pc}}} \sqrt{\frac{\pi}{24.5}} \frac{1}{E_{\mathrm{r}}}$,

where $E_{\mathrm{r}}$ represents the biaxial reduced modulus

$\frac{1}{E_{\mathrm{r}}}=\frac{1-v^{2}}{E}+\frac{1-v_{\mathrm{i}}^{2}}{E_{\mathrm{i}}}$.

In Eq. (4), $E$ and $v$ stand for Young's modulus and Poisson's ratio $(v=0.3)$ for the film, respectively. $E_{\mathrm{i}}$ and $v_{\mathrm{i}}$ denote the same quantities for the diamond indenter $\left(E_{\mathrm{i}}=1050 \mathrm{GPa}, v_{\mathrm{i}}=0.07\right)$. This leads to the final equation, which calculates the values of Young's modulus for the coating

$E=\frac{\left(1-v^{2}\right) \sqrt{\pi / 24.5}}{2 h_{\mathrm{pc}}(\mathrm{d} h / \mathrm{d} p)-\left(\left(1-v_{i}^{2}\right) / E_{\mathrm{i}}\right) \sqrt{\pi / 24.5}}$.

\subsection{Scratch tests}

An established method of assessing the adhesion of hard coatings made by PVD is the scratch test. During this process, a hemispherical tipped diamond indenter is driven over the coated surface to produce a scratch. The equipment used was a Sebastian-5A model from the Quad Group. A $200 \mu \mathrm{m}$ radius diamond tip was used for the stylus; the test indentation speed was set at $0.01 \mathrm{~cm} / \mathrm{s}$, and the load rate was kept at $1.2 \mathrm{~N} / \mathrm{s}$. The load on the diamond stylus is increased linearly whilst at the same time the scratch length is being monitored.

A selective and useful criteria based on acoustic emission (AE) is employed. This AE signal is defined as the elastic wave generated by the release of energy stored within the material structure [9]. An AE transducer with a resonance frequency of $200 \mathrm{kHz}$, which can detect acoustic bulk waves in a frequency range of approximately $50-400 \mathrm{kHz}$ (insensitive to mechanical vibrations arisen from instruments: 0$30 \mathrm{kHz}$ ), was used. Due to the fact that the AE signal contains information as to the extent of the damage (released binding energy), its signal intensity is integrated with respect to time and subsequently transformed to an energy spectrum. The analysis of this AE spectrum aids the identification of the value of the critical load.

The load at which the first adhesion failure occurs is known as the first critical load $\left(L_{\mathrm{c} 1}\right)$ [9]. Frequently this first critical load is associated with a cohesive failure related to chipping of material from within the coating. This damage is representative of a very weak AE signal and practically goes unnoticed in the frictional transversal force versus scratch length plot. Nevertheless, an experienced observer using an optical microscope can detect this failure easily. 
The critical load characteristic of total failure of the coating $\left(L_{\mathrm{c} 2}\right)$ is regarded as the inflection point of the increasing frictional force curve. This point corresponds to the first contact of the diamond with the substrate, resulting in a change of the shear stress component [9].

The mechanisms that give rise to adhesion failures are regarded as cohesive or adhesive damage. When chipping occurs at some particular distance preceding the indenter it can result in buckling and cracking in a roughly semicircular arc pointing towards the scratch direction [10]. The sliding stylus produces a compressive stress field ahead, which deforms the coating, bends it and pulls it underneath, embedding the chipped material in the scratch track. When buckling is the origin of total failure, the corresponding critical load $\left(L_{\mathrm{c} 2}\right)$ is low. Contrarily, if the mechanism that gives rise to total failure is related to spallation, then the critical load is higher. Spallation is an adhesive damage, and occurs when there is flaking of coating material from its interface with the substrate, being deposited subsequently on the lateral top edge of the film. This effect generates a considerable AE signal, making its detection normally very easy.

Two other types of failure mechanisms can be linked to adhesion damage. Both happen when the coating remains fully adherent to the substrate [10]. The first type is named conformal cracking, and consists of semi-circular cracks within the scratch only, parallel to the leading edge of the indenter. They are formed as the indenter deforms the coating and underlying substrate, resulting in tensile bending moments within the coating as it is pushed down underneath the indenter. The second type bears resemblance to the former in the way that there is also a semi-circular arc in the scratch track, although these traces are now parallel to the trailing edge of the stylus. The latter result from tensile frictional stresses presents on the trailing edge of the indenter; these stresses balancing the compressive stresses ahead of the indenter.

Associated with these failure mechanisms, quite often lateral cracking can be observed also. At low critical loads, this lateral cracking reaches out from the semi-circular arcs resulting from chipping and buckling. At higher critical loads, when the tensile compressive field that follows the trailing edge of the indenter is considerable in respect to the compressive fields ahead of the stylus, lateral-cracking converging to the opposite direction of the leading stylus takes place.

\subsection{Residual stress analysis}

The technique used for residual-stress measurement is based on the associated curvature deflection of a thin substrate $[11,12]$. The major advantages of this approach in comparison to the measurements of the elastic strains in the films via XRD [13] resides in the fact that in the deflection method no information regarding the elastic properties of the coating is required to calculate the residual stress.
It is well known that both the mechanical and tribological properties of coated specimens are affected by the size and relative spatial distribution of residual stresses [14]. An example of this is that a high compressive stress within the coating has been found to increase its fracture toughness [15]. High coating toughness is a control quality sought after in PVD coatings applied to tool steels, where wear is often induced by propagation of cracks within the coating. However, excessive compressive stresses have the disadvantage of provoking adhesion damage to the coatings, mainly around the edges.

Experimentally, this technique works well when the thickness of the substrate exceeds the film thickness by at least a factor of 100, and the lateral dimensions of the coating and substrate are much greater than their total thickness. In PVD coatings there is usually a large intrinsic (microstructural) component in the residual stress $\left(\sigma_{\text {res }}\right)$, which originates: partly by the way that the crystal structure defines the growth of the deposit, through changes in the distribution, orientation and size of the crystallites nucleated; and partly by the PVD processes itself [16].

Using the Stoney [17] equation

$\sigma=-\frac{E_{\mathrm{s}}}{6\left(1-v_{\mathrm{s}}\right)} \times \frac{t_{\mathrm{s}}^{2}}{t_{\mathrm{c}}} \times\left[r_{\mathrm{a}}^{-1}-r_{\mathrm{b}}^{-1}\right]$,

in which $E_{\mathrm{S}} /\left(1-v_{\mathrm{S}}\right)$ refers to the biaxial modulus of the substrate, where $E_{\mathrm{s}}=2150 \mathrm{GPa}$ and $v_{\mathrm{s}}=0.283 \quad[20] ; t_{\mathrm{s}}$ and $t_{\mathrm{c}}$ are, respectively, the thickness of the steel substrate and coating; and the parameters $r_{\mathrm{b}}$ and $r_{\mathrm{a}}$ represent the radius of the curvature of the substrate before and after deposition.

The samples used were deposits of TiN/ZrN multi-layers on $25 \mathrm{~mm}$ diameter and $500 \mu \mathrm{m}$ thick stainless steel discs. Using a Keyence 2100 laser displacement meter coupled with a semiconductor laser, it was possible to measure the deflection of the substrates prior to and after deposition. The theory developed by Ramsey et al. [18] was used to obtain a value for the radius of curvature of the discs. A parabolic equation

$\delta(x)=a+b x+c x^{2}$

was fitted to the experimental deflection curves, before and after deposition. $\delta(x)$ is the deflection as a function of the lateral sample length; whilst $a, b$ and $c$ are constants. From the latter equation, the radius of curvature is easily assessed as

$r=(-2 C)^{-1}$.

\section{Experimental results}

\subsection{Hardness and Young's modulus}

Table 1 shows the experimental results obtained for the ultra-microhardness of the TiN/ZrN coatings. $P_{\max }$ is the 
Table 1

Ultra-microhardness and Young's modulus values obtained for TiN/ZrN multi-layers

\begin{tabular}{llcll}
\hline Sample & $t_{\mathrm{c}}(\mu \mathrm{m})$ & $P_{\max }(\mathrm{mN})$ & $E(\mathrm{GPa})$ & $\mathrm{HV}\left(\mathrm{kg} / \mathrm{mm}^{2}\right)$ \\
\hline M14 & 0.95 & 30 & $228 \pm 31$ & $1330 \pm 347$ \\
M16 & 1.50 & 50 & $283 \pm 6$ & $1998 \pm 110$ \\
M17 & 1.14 & 30 & $273 \pm 7$ & $2132 \pm 170$ \\
M18 & 1.10 & 30 & $322 \pm 28$ & $2779 \pm 347$ \\
M20 & 1.86 & 100 & $318 \pm 32$ & $3583 \pm 978$ \\
M21 & 1.91 & 50 & $370 \pm 31$ & $3631 \pm 381$ \\
M22 & 1.81 & 70 & $244 \pm 58$ & $1889 \pm 534$ \\
M31 & 1.89 & 70 & $329 \pm 13$ & $3060 \pm 170$ \\
\hline
\end{tabular}

maximum load employed during the indentation cycle; $\mathrm{HV}$ and $E$ are the Vickers ultra-microhardness and Young's modulus, respectively. $P_{\max }$ was chosen taking into account the coating thickness, in order to eliminate possible substrate influence on the final results. An average number of 6 tests was performed on each sample. Samples M16, M18 and M21 were produced in the rotation mode whilst the remaining samples were in the static mode. In this table $t_{\mathrm{c}}$ represents the coating thickness.

In Fig. 1 are presented the results of an indentation test performed on a sample (M21), in order to gather data related to its ultra-microhardness.

Fig. 2 illustrates the evolution of both the ultra-microhardness values and Young's modulus, relative to the multilayer modulation periodicity, for TiN/ZrN multi-layer samples grown via: (a) rotation; and (b) static; modes. Error bars are given for the sake of clarity. The bilayer period was determined from structural refinement of the XRD high angle patterns of the same films [19].

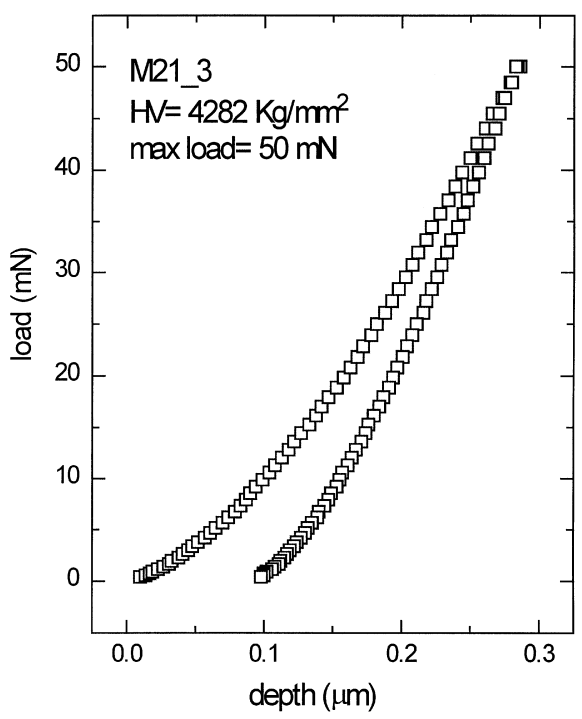

Fig. 1. Ultra-microhardness test performed on a sample (M21) grown in the rotation mode. The maximum load was set at $50 \mathrm{mN}$. The creep time, at the final stage of loading, was held for $32 \mathrm{~s}$.

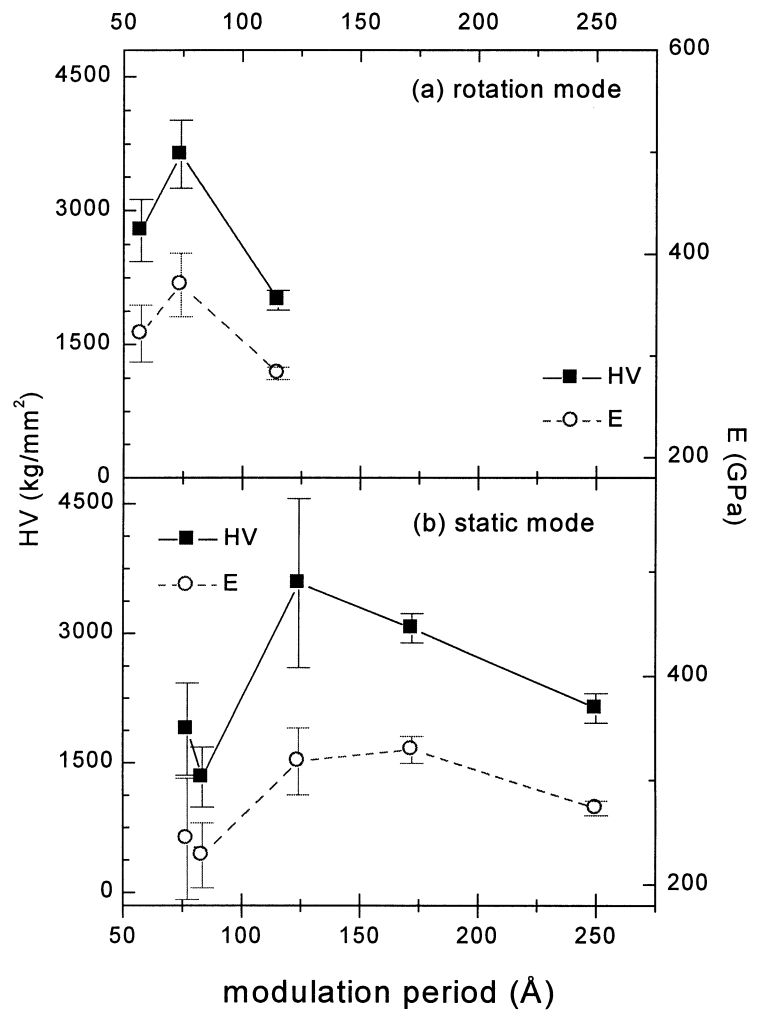

Fig. 2. Evolution of $\mathrm{HV}$ and $E$ relative to the modulation period of the TiN/ZrN films grown by: (a) rotation; and (b) static modes. The periodicity was calculated by XRD refinement [19].

\subsection{Adhesion tests}

Table 2 presents a summary of all of the experimental data extracted for a series of samples regarding the critical loads detected for the first failure $\left(L_{\mathrm{c} 1}\right)$ and the total adhesion failure $\left(L_{\mathrm{c} 2}\right)$.

Fig. 3 represents an example of a scratch test performed on the sample M10, grown by static mode. Visible are the plots of the AE signal, the transversal frictional force and

Table 2

Critical load values obtained for the first failure $\left(L_{\mathrm{c} 1}\right)$ and total failure $\left(L_{\mathrm{c} 2}\right)$ corresponding to TiN/ZrN multi-layers, when subjected to a scratch test

\begin{tabular}{lrll}
\hline Sample & $\Delta(\AA)$ & $L_{\mathrm{c} 1}(\mathrm{~N})$ & $L_{\mathrm{c} 2}(\mathrm{~N})$ \\
\hline M10 & 1000 & $15 \pm 5$ & $45 \pm 2$ \\
M12 & 395 & $13 \pm 2$ & $31 \pm 2$ \\
M13 & 124 & $10 \pm 1$ & $20 \pm 2$ \\
M15 & 82 & $10 \pm 1$ & $20 \pm 1$ \\
M16 & 115 & $19 \pm 6$ & $31 \pm 3$ \\
M17 & 250 & $11 \pm 5$ & $23 \pm 5$ \\
M18 & 57 & $16 \pm 2$ & $29 \pm 8$ \\
M20 & 124 & $16 \pm 5$ & $26 \pm 6$ \\
M21 & 74 & $9 \pm 2$ & $32 \pm 2$ \\
M22 & 77 & $10 \pm 2$ & $20 \pm 4$ \\
M31 & 172 & $24 \pm 4$ & $34 \pm 5$ \\
M40 & 124 & $12 \pm 2$ & $35 \pm 6$ \\
\hline
\end{tabular}

The modulation period $(\Delta)$ is also shown [19]. An average number of 6 tests was performed on each sample. 


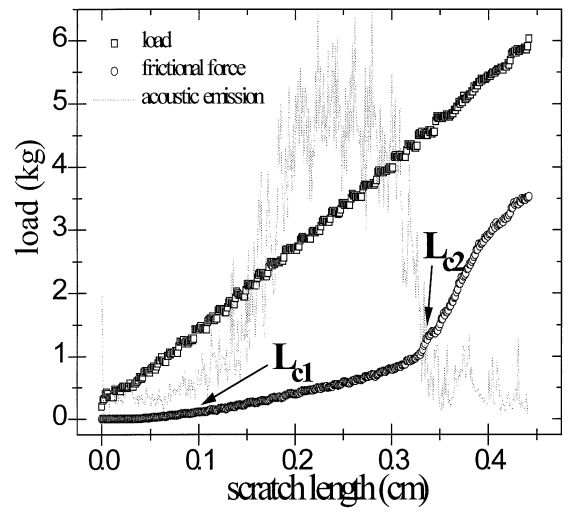

Fig. 3. Scratch test performed on a TiN/ZrN multi-layer (M10) with a maximum load of $6 \mathrm{~kg} . L_{\mathrm{c} 1}$ is indicative of chipping from within the coating, and $L_{\mathrm{c} 2}$ corresponds to the total failure derived from extensive spallation.

load versus scratch length. $L_{\mathrm{c} 1}$ and $L_{\mathrm{c} 2}$ are clearly marked, and were assessed by combined optical and plot analysis. The first critical load, when observing the damage optically, was associated with chipping. The second critical load was linked to an extensive spalling that occurred and lead to total adhesion failure. Whilst observing Fig. 3, it is easy to point out where this last load occurred. First, if the frictional force plot versus scratch length is examined, there is a slight perturbed inflection point indicative of this failure. Besides this, experimentally it is found that $L_{\mathrm{c} 2}$ is directly related to the abrupt decay of the acoustic emission signal. Nevertheless, the optical observation and position detection of the damage due to $L_{\mathrm{c} 2}$ is essential. A rule of thumb, while observing the damage optically, dictates the position of $L_{\mathrm{c} 2}$ to where there is approximately less than $50 \%$ of the coating remaining. Only then and based on the observations of the frictional force plots and AE signal, can the second critical load be derived.

For the scratch test illustrated in Fig. 3, despite the second critical load being identified at a position $\sim 0.34 \mathrm{~cm}$ on the scratch length, the coating is only removed completely at around $\sim 0.4 \mathrm{~cm}$. This is clearly seen on the frictional force plot, where an inflection point occurs. On the scratch tracks, occasionally, appear some stains that are due to substrate roughness. These roughness peaks protrude through the coating.

If attention is directed to Fig. 4(a), some information can be seen regarding the most common mechanism, which originates total failure in the samples credited with a low critical load. This is relative to sample M13, grown by the static mode. Clearly visible is a semi-circular arcs resulted from chipping and buckling embedded on the track. Lateral cracking progressively shows, as adhesion failure is eminent.

Fig. 4(b) presents another photograph obtained by optical observation of sample M16 grown by rotation. This time, the failure mechanism that dominated the total failure in the vicinity of $L_{\mathrm{c} 2}$ is associated with tensile cracking within the (a)

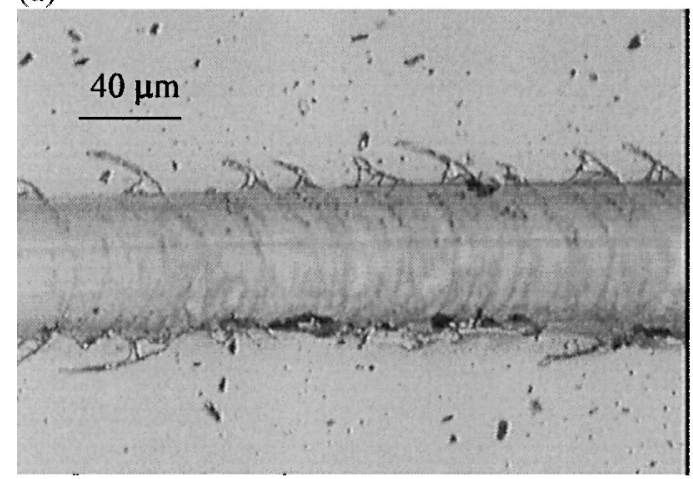

(b)

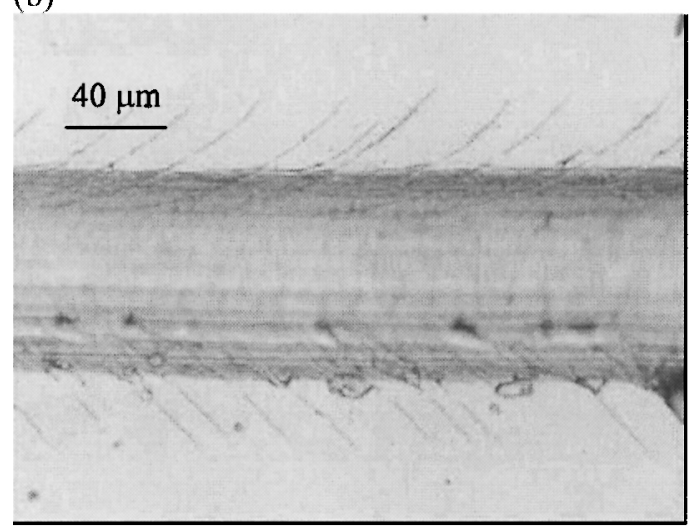

(c)

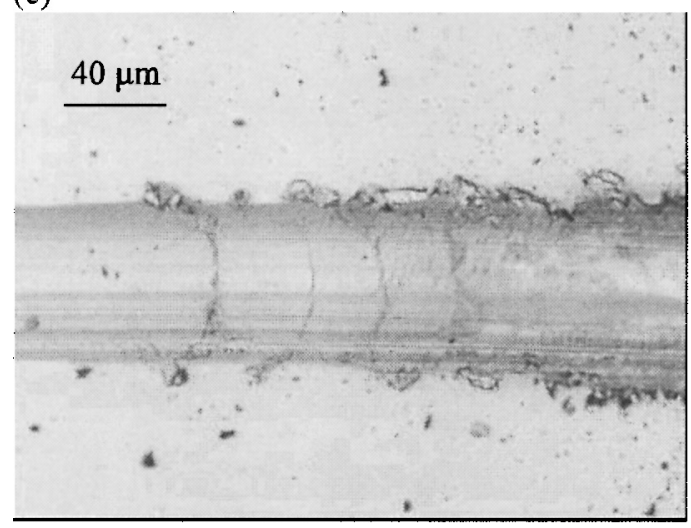

Fig. 4. Failure mechanisms exhibited in TiN/ZrN multi-layer samples: (a) buckling and chipping in M13; (b) tensile cracking in M16; (c) spallation in M40.

scratch track and tensile lateral cracking. Fig. 4(c) corresponds to sample M40 grown in the static mode. This sample exhibits one of the highest total failure critical loads achieved $(35 \mathrm{~N})$, from the scratch tests performed on the TiN/ZrN multi-layer coatings. In all the samples with critical loads of this order of magnitude the mechanism that is related to total failure is spallation. Conformal cracking is also observable immediately prior to the final damage. In all the scratch tests shown in Fig. 4, the testing itself proceeds to the right.

Fig. 5 elucidates the behaviour of $L_{\mathrm{c} 1}$ and $L_{\mathrm{c} 2}$ versus the TiN/ZrN modulation periodicity regarding samples grown by: (a) the rotation mode; and (b) the static mode. Fig. 5(c) 


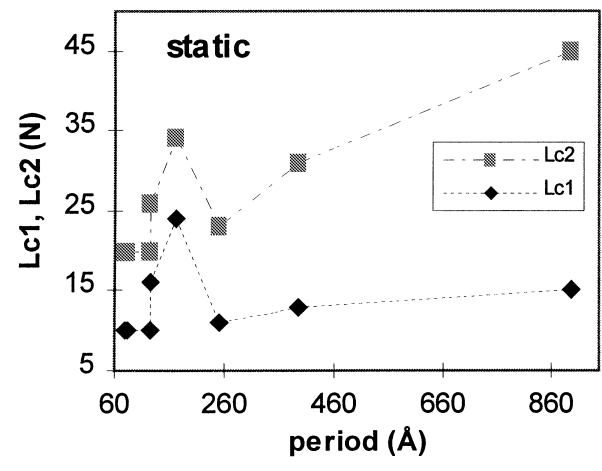

(a)

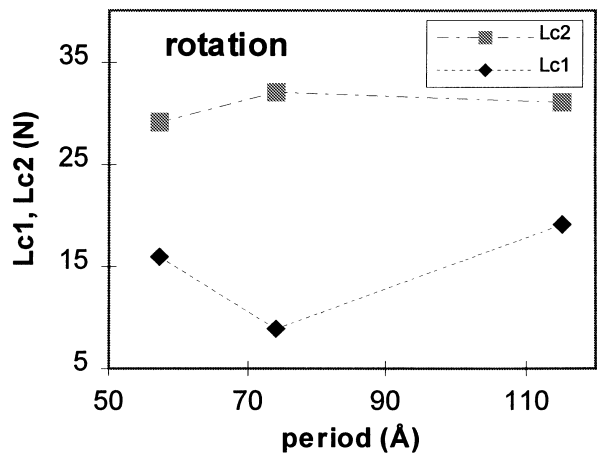

(b)

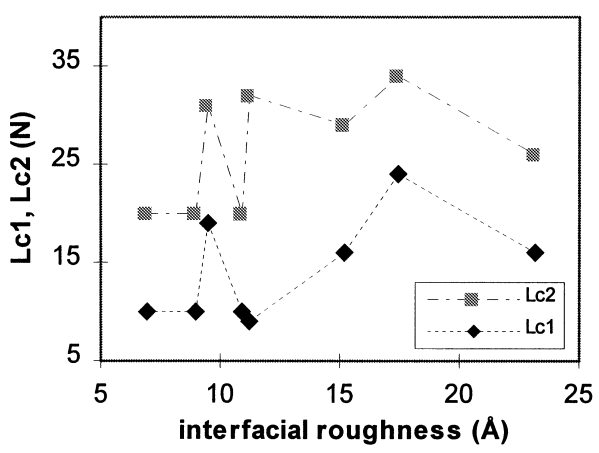

(c)

Fig. 5. Evolution of $L_{\mathrm{c} 1}$ and $L_{\mathrm{c} 2}$ critical loads in respect to the modulation period for TiN/ZrN multi-layer samples grown via: (a) the rotation mode; and (b) the static mode. Their behaviour according to the (c) interfacial roughness [19] present on the coatings is also shown.

refers to the evolution of both critical loads, this time with respect to the total interfacial roughness. This roughness was calculated by structural computational refinement of the XRD spectra, relative to these samples [19].

\subsection{Residual stress analysis}

In Table 3 the experimental data obtained for the residual stress analysis of TiN/ZrN multi-layer coatings can be seen. In this table $t_{\mathrm{c}}$ represents the coating thickness; $\Delta$ stands for the multi-layer period, which was calculated by structural refinement of the XRD patterns [19]; $r_{\mathrm{b}}$ and $r_{\mathrm{a}}$ represent the curvature radius of the samples, before and after deposition,

Table 3

Experimental compressive residual stress values obtained for a series of studied TiN/ZrN multi-layer samples

\begin{tabular}{lllll}
\hline Sample & $t_{\mathrm{c}}(\mu \mathrm{m})$ & $\Delta(\AA)$ & $1 / r_{\mathrm{a}}-1 / r_{\mathrm{b}}\left(\mathrm{m}^{-1}\right)$ & $\sigma_{\text {res }}(\mathrm{GPa})$ \\
\hline M20 & 1.86 & 124 & 0.987 & 6.64 \\
M21 & 1.91 & 74 & 0.232 & 1.52 \\
M22 & 1.81 & 124 & 0.330 & 2.28 \\
M23 & 2.17 & 90 & 0.636 & 3.67 \\
M24 & 0.71 & 124 & 0.434 & 7.70 \\
M26 & 1.84 & - & 4.790 & 3.26 \\
M31 & 1.89 & 172 & 1.180 & 7.81 \\
M32 & 1.75 & 140 & 1.060 & 7.58 \\
M34 & 4.02 & - & 0.120 & 3.73 \\
\hline
\end{tabular}

respectively; and $\sigma_{\text {res }}$ is the residual stress. All values of $\sigma_{\text {res }}$ are indicative of compressive residual stresses within the coatings.

Samples M21, M23 and M32 were deposited via the rotation mode. Samples M26 and M34 refer not to multilayers but to thin films of $\mathrm{TiN}$ and $\mathrm{ZrN}$, respectively. Curiously, both exhibit compressive residual stresses of the order of $\sim 3.5 \mathrm{GPa}$. The remaining samples were grown under the static mode.

Fig. 6 demonstrates the columnar growth characteristic of these coatings. The sample shown corresponds to M30, which was grown in the rotation deposition mode.

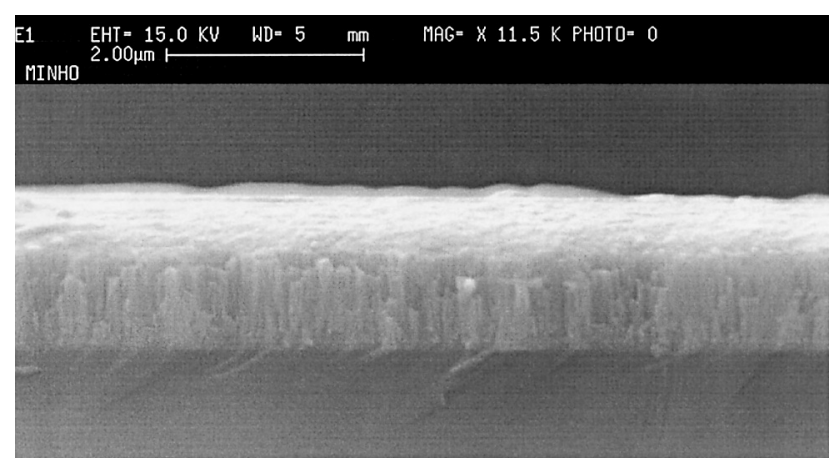

Fig. 6. SEM photograph of sample (M30-rotation), exhibiting dense columnar morphology. This growth is typical of TiN/ZrN multi-layers. 


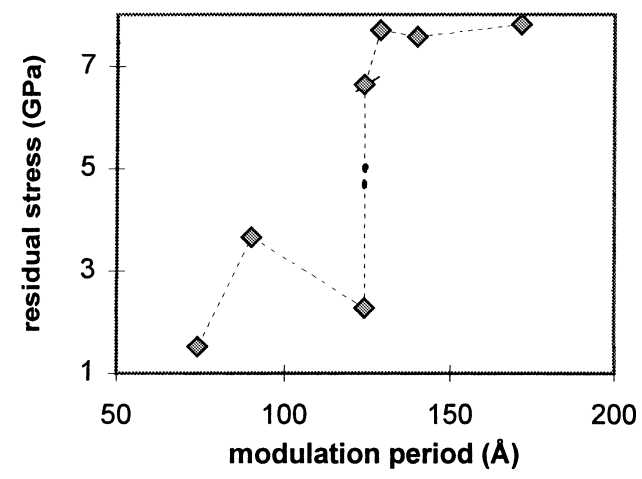

Fig. 7. Behaviour of the residual stress in respect to the bi-layer period, for a series of samples. All stresses are compressive.

Fig. 7 illustrates the behaviour of the residual stress versus the TiN/ZrN multi-layer modulation period. This periodicity was determined by structural simulation of the experimental XRD patterns of the samples [19].

\section{Conclusions}

Reported in this paper is the production of TiN/ZrN PVD multi-layers. Whilst studying the ultra-microhardness and Young's modulus data obtained for the TiN/ZrN multi-layer coatings, it is possible to comment that there is a similar behaviour between both; regarding essentially their evolution versus number of bi-layers, modulation period, total interfacial roughness and film thickness. A slight increase on both $\mathrm{HV}$ and $E$ with modulation period is observed, although some attention has to be reserved towards the overall film thickness. The samples created under rotation exhibited larger values of interfacial roughness, which influenced positively $\mathrm{HV}$ and $E$; rewarding these samples with better ultra-microhardness and Young's modulus levels in comparison with similar multi-layers grown in the static mode. Ultra-microhardness values as high as $~ 3600$ Vickers were obtained, which is optimistic for industrial applications, more precisely in wear prevention. The elastic Young's modulus values are appreciable, in a range between 230 and $370 \mathrm{GPa}$; being a little lower than those published for thin films of TiN ( 450 GPa [11]).

There is a link between $\mathrm{HV}, E$ and $L_{\mathrm{c} 2}$ values. For a large portion of samples, higher values of ultra-microhardness and Young's modulus corresponded to higher levels of total failure critical loads.

The adhesion tests indicated the mechanisms that induced failures. Through all of the experiments fulfilled, it can be concluded that for lower critical loads $(\sim 20 \mathrm{~N})$ the mechanism involved is of a cohesive nature: chipping plus buckling. At intermediate levels of $L_{\mathrm{c} 2}(\sim 30 \mathrm{~N})$ tensile cracking is present, whilst at higher magnitudes of this critical load $(\sim 32-45 \mathrm{~N})$ the failure that dominates the total adhesion damage of the coating is directly linked to spallation. Lateral cracking is also evident, being compressive at lower levels of $L_{\mathrm{c} 2}$ whilst at higher values it has a tensile nature.

Samples deposited under the rotation mode exhibited similar critical loads and shared the same failure mechanisms, which enabled a more consistent study to be made than grown under static conditions.

Concerning the residual stress measurements, there is a visible increment with the decrease of the number of bilayers, and consequent monolayer thickness. This means that $\sigma_{\text {res }}$ increases with the thickness of a bi-layer (period), when comparing samples with approximately the same overall film thickness. At some critical stage in the fabrication of a bi-layer, the residual stress reaches an elevated level that causes inevitable brittleness and consequently lower adhesion critical loads. All of the samples were characteristic of a compressive nature having residual stresses of between 1 and $8 \mathrm{GPa}$. The majority of these stress results for TiN/ZrN PVD multi-layers are much higher than those found in the literature for PVD thin films of TiN $(\sim 4.0 \mathrm{GPa}[11])$.

\section{Acknowledgements}

The authors gratefully acknowledge the financial support of the Junta Nacional de Investigação Científica (JNICT), during the course of this scientific research, under the project referenced as PBICT/P/CTM/1962/95.

\section{References}

[1] R. Fella et al., Surf. Coat. Tech. 36 (1988) 257.

[2] R. Schlatmann et al., Phys. Rev. B. 51 (1991) 5345.

[3] K.J. Ma et al., Surf. Coat. Tech. 76-77 (1995) 297.

[4] W.-D. Munz et al., Surf. Coat. Tech. 58 (1993) 205.

[5] M. Shinn et al., J. Mater. Res. 7 (1992) 901.

[6] X. Chu et al., Surf. Coat. Tech. 61 (1993) 251.

[7] G.M. Pharr, W.C. Oliver, MRS Bull. (1992) 28.

[8] W.C. Oliver, G.M. Pharr et al., J. Mater. Res. 7(6) (1992) 1564.

[9] V. Bellido-Gonzallez et al., Surf. Coat. Tech. 74 (1995) 884.

[10] P.J. Burnett et al., Thin Sol. Films 154 (1987) 403.

[11] M. Larsson et al., Surf. Eng. 12(1) (1996) 43.

[12] H.Y. Kumagai, Ninth International Conference on CVD, The Electrochem. Soc., 1984, p. 189.

[13] A.J. Perry, J. Vac. Sci. Tech. A 8(3) (1990) 1351.

[14] W.D. Nix, Metall. Trans. 20A (1989) 2225.

[15] G. Kleer et al., Surf. Coat. Technol. 54-55 (1992) 165.

[16] J.-Å. Schweitz, J. Micromech. Microeng. 1 (1991) 10.

[17] G.G. Stoney, Proc. R. Soc. London, Ser. A 82 (1909) 172.

[18] P.M. Ramsey et al., Surf. Coat. Tech. 43-44 (1990) 223.

[19] C.J. Tavares et al., Surf. Coat. Tech. 100-101 (1998) 65.

[20] C.J. Smithels, Metals Reference Book, 5th ed., Butterworth, London, 1976, p. 975. 